\title{
Stretch marks during pregnancy: a review of topical prevention*
}

\author{
K. Korgavkar ${ }^{1}$ and F. Wang ${ }^{2}$ \\ ${ }^{1}$ Medical School and ${ }^{2}$ Department of Dermatology, University of Michigan, 1910 Taubman Center, 1500 East Medical Center Drive, Ann Arbor, MI 48109, \\ U.S.A.
}

\section{Summary}

\section{Correspondence \\ Frank Wang. \\ E-mail: frawang@med.umich.edu}

\section{Accepted for publication \\ 17 September 2014}

\section{Funding sources}

None.

\section{Conflicts of interest}

None declared.

*Plain language summary available online

DOI $10.1111 /$ bjd.13426
Striae gravidarum (SG), or stretch marks developing during pregnancy, affect up to $90 \%$ of women. While not medically dangerous, SG can be disfiguring, causing emotional and psychological distress. However, studies specifically addressing the prevention of SG, especially during pregnancy, are sparse. Furthermore, the molecular pathogenesis of SG is unclear and may differ from that of striae from other causes. Considering these factors, we review topical modalities that have been used specifically for preventing SG during pregnancy. We identify two major strategies (end points) addressed by these modalities, namely (i) preventing the de novo development of SG and (ii) reducing the severity of SG that have recently developed. We also identify risk factors for the development of SG and suggest that pregnant women with these risk factors are an appropriate target population for prevention. In reviewing the literature, we find that there is limited evidence that centella, and possibly massage with bitter almond oil, may prevent SG and/or reduce their severity. There is weak evidence that hyaluronic acid prevents SG. Tretinoin holds promise for reducing the severity of new-onset SG, but its use is limited by its pregnancy category. Finally, cocoa butter and olive oil are not effective for preventing SG or reducing the severity of lesions. We conclude that reliable methods for preventing SG are scarce. Furthermore, available topical modalities generally lack strong evidence from rigorous, welldesigned, randomized controlled trials with ample numbers of subjects. Thus, further research is necessary to elucidate SG pathogenesis, which may lead to effective prevention modalities.

\section{What's already known about this topic?}

- Striae gravidarum (SG), or stretch marks developing during pregnancy, can be disfiguring, causing emotional and psychological distress.

- Studies specifically addressing the prevention of SG during pregnancy are sparse.

\section{What does this study add?}

- We review topical modalities that have been used specifically for preventing SG during pregnancy.

- We conclude that reliable methods for preventing SG are scarce, and that topical modalities generally lack strong evidence from rigorous, well-designed trials with ample numbers of subjects.
The term striae distensae (SD) refers to stretch marks from various causes, including pregnancy, weight gain, obesity, growth spurts and corticosteroid excess. ${ }^{1-3}$ Striae gravidarum (SG) are stretch marks specifically developing during pregnancy. SG affect up to $90 \%$ of women. ${ }^{4}$ They appear in the second or third trimester, typically on the abdomen and breasts, and less commonly on the buttocks, hips and thighs. ${ }^{2}$ Initially, lesions are pink to violaceous and may be oedematous 
or pruritic (striae rubra). Over months to years, the lesions mature into white, shiny, atrophic, crinkly streaks that are permanent (striae alba).

Although SG are common, their pathogenesis remains unclear. Mechanical tension may play a pathogenic role by damaging dermal connective tissue, as the long axis of lesions is usually oriented perpendicularly to the direction of greatest skin stretching. ${ }^{3,5}$ However, this possibility remains controversial, as increased maternal weight gain during pregnancy, baseline maternal body mass index and infant birthweight are not consistently associated with SG development. ${ }^{3,4,6,7}$

At the molecular level, disruption of dermal connective tissue, including collagen fibrils and elastic fibres, likely leads to decreased strength and elasticity of lesions., ${ }^{5,8}$ Indeed, early, erythematous SG exhibit focal loss and thinning of collagen bundles. ${ }^{8,9}$ Variable reduction, thinning and disorganization of elastic fibres are seen at this stage and may result from elastolysis mediated by infiltrating mast cells and macrophages. ${ }^{6,8-10}$ Additionally, elastic fibre disorganization may result, in part, from reduction of fibrillin microfibrils in early SG. ${ }^{9}$ Increased hormones during pregnancy, such as glucocorticoids, may suppress fibroblast production of collagen fibrils and elastic fibres, thereby hindering complete 'repair' of disrupted connective tissue. ${ }^{1,3}$

In mature SG, the synthetic capacity of fibroblasts remains reduced, which likely promotes persistence of disrupted connective tissue components and, hence, permanency of lesions. ${ }^{11}$ Histologically, mature SG can resemble scars, with collagen bundles and elastic fibres appearing thin or decreased, and oriented parallel to the epidermis, which appears atrophic. $^{6,8,9,11}$

While not medically dangerous, SG are disfiguring, causing emotional and psychological distress. ${ }^{2,12}$ Thus, women remain interested in preventing SG and/or reducing their severity. However, most published studies address the treatment of established SD from a variety of causes. Studies specifically addressing the prevention of new-onset SG or reduction of their severity during pregnancy are sparse. Considering that the molecular pathogenesis of SG may differ from that of SD from other causes, ${ }^{13,14}$ we focus specifically on SG in this review. Our review differs from recently published ones ${ }^{3}$ in that we discuss SG apart from other types of SD and limit our analysis to topical agents that have been used specifically in the pregnant population.

Additionally, we identify two major strategies (end points) for the prevention of SG, namely (i) preventing the de novo development of SG and (ii) reducing the severity of SG that have recently developed. To provide clinicians with an evidence-based review aimed at pregnant women, this report discusses topical modalities with these end points in mind (Table 1).

\section{Methods}

A systematic PubMed and Google Scholar search was performed using the terms 'striae', 'striae gravidarum', 'stretch marks', 'pregnancy', 'lifestyle', 'diet', 'exercise', 'centella', 'Trofolastin', 'oil', 'cocoa butter', 'massage', 'hyaluronic acid', 'Alphastria', 'Verum' and 'tretinoin'. Review articles, observational studies, case series and cohort studies published from 1950 to 2014 were included. Articles published in non-English languages that were able to be translated were included. The following article types were excluded: conference proceedings, abstracts, and publications in non-English languages that were unable to be translated.

\section{Risk factors}

An important aspect of SG prevention is predicting their development, so that lifestyle modifications or prophylactic therapies can be initiated. Identifying risk factors is central to this goal, but recognizing those consistently associated with SG development remains challenging. Pregnant women with one or more of the attributes listed in Table 2 could be at increased risk for developing SG and would be an appropriate target population for prevention strategies. ${ }^{4,14-23}$

\section{Lifestyle modifications}

- Evidence for diet and exercise in preventing SG is lacking.

As indicated in Table 2, increased maternal body mass index and weight gain during pregnancy may be associated with SG development. Accordingly, a healthy diet and regular exercise may play a role in SG prevention. Indeed, some clinicians suggest that stretching exercises, such as aerobics, may be beneficial in this regard. However, there is a lack of data supporting the efficacy of lifestyle modifications in preventing SG or reducing their severity during pregnancy. In one study of 80 nonpregnant individuals, $79 \%$ of whom had SD, a $3-$ month weight-loss programme did not improve the severity of lesions, regardless of the intervention (diet alone, diet plus aerobic exercise, or diet plus resistance exercise). ${ }^{24,25}$

\section{Topical modalities for preventing striae gravidarum}

\section{Centella}

- Creams containing centella, a medicinal herb, may be effective for preventing SG and reducing their severity.

- As centella is often combined with other ingredients, its specific role in SG prevention requires further investigation.

Centella asiatica, commonly known as centella, is a plant found in South Asia that has been studied for treating leprosy, lupus, venous stasis ulcers and eczema. ${ }^{26}$ Trofolastin is a proprietary product containing centella extract, $\boldsymbol{\alpha}$-tocopherol and collagen-elastin hydrolysates. Another proprietary product contains centella triterpenes formulated in a cream with hydroxyprolisilane-C, rosehip oil and vitamin E. While the mechanism of action of centella is unclear, this ingredient may stimulate fibroblasts and inhibit glucocorticoid activity. ${ }^{27,28}$ 


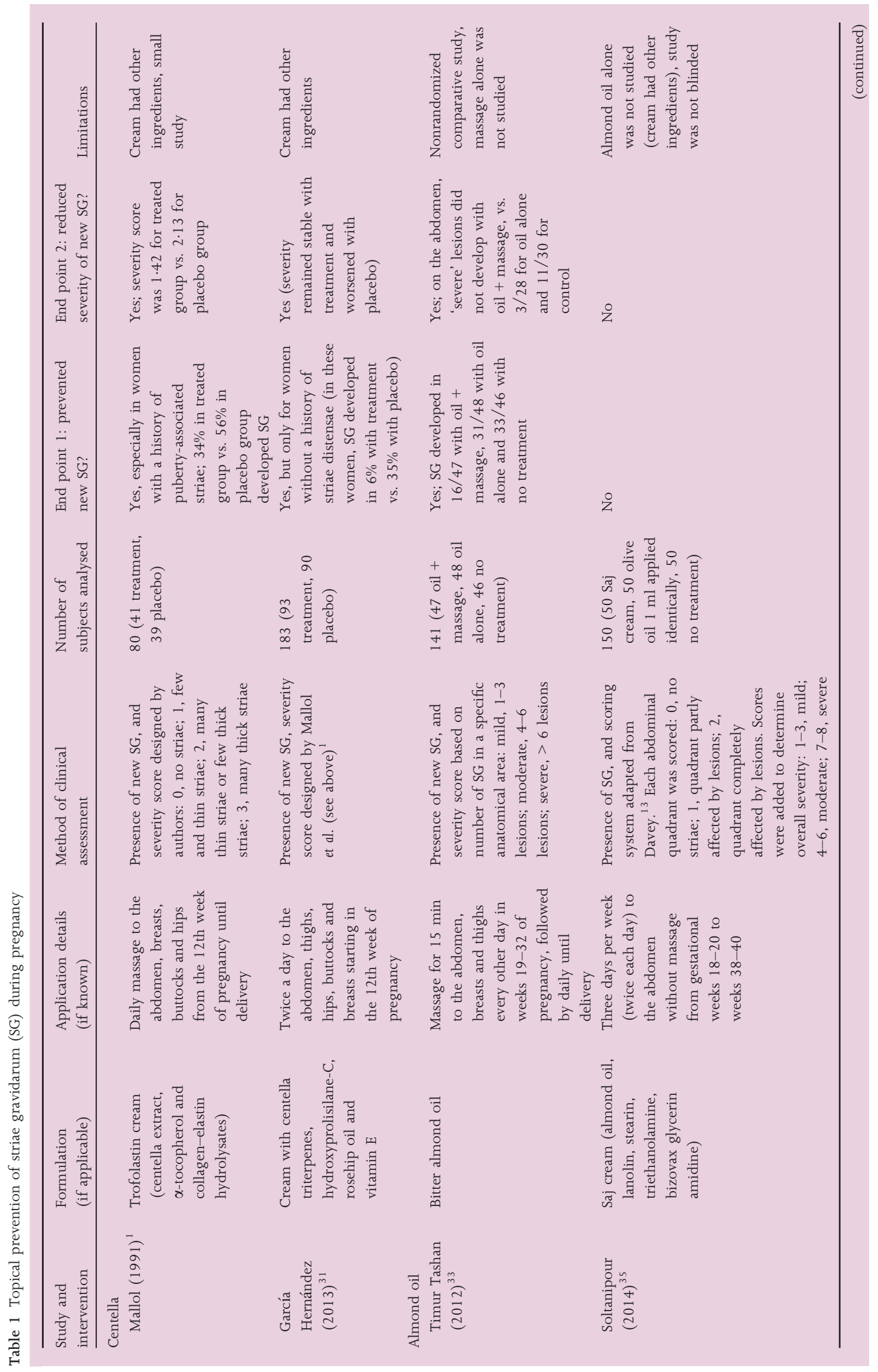




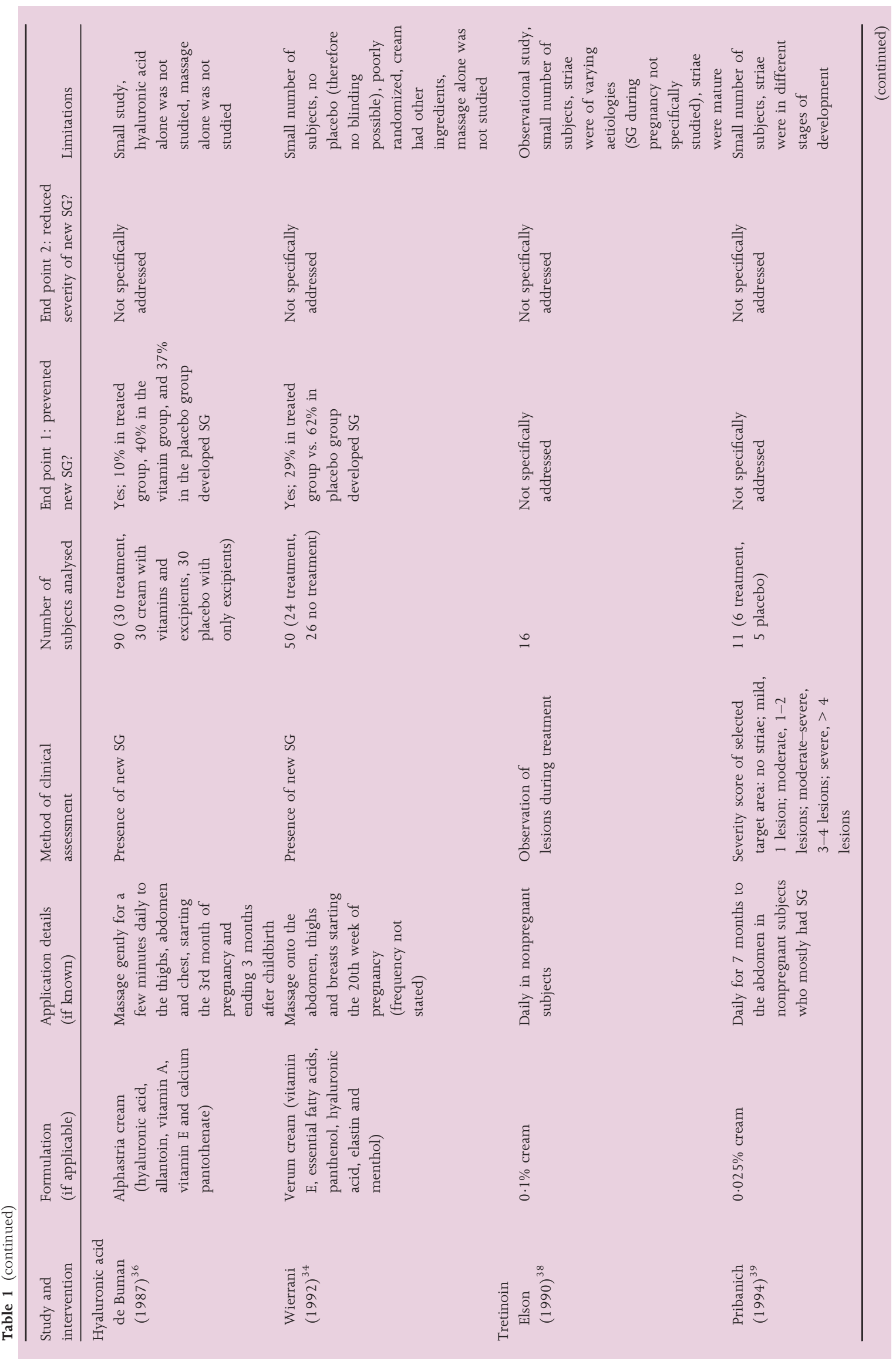




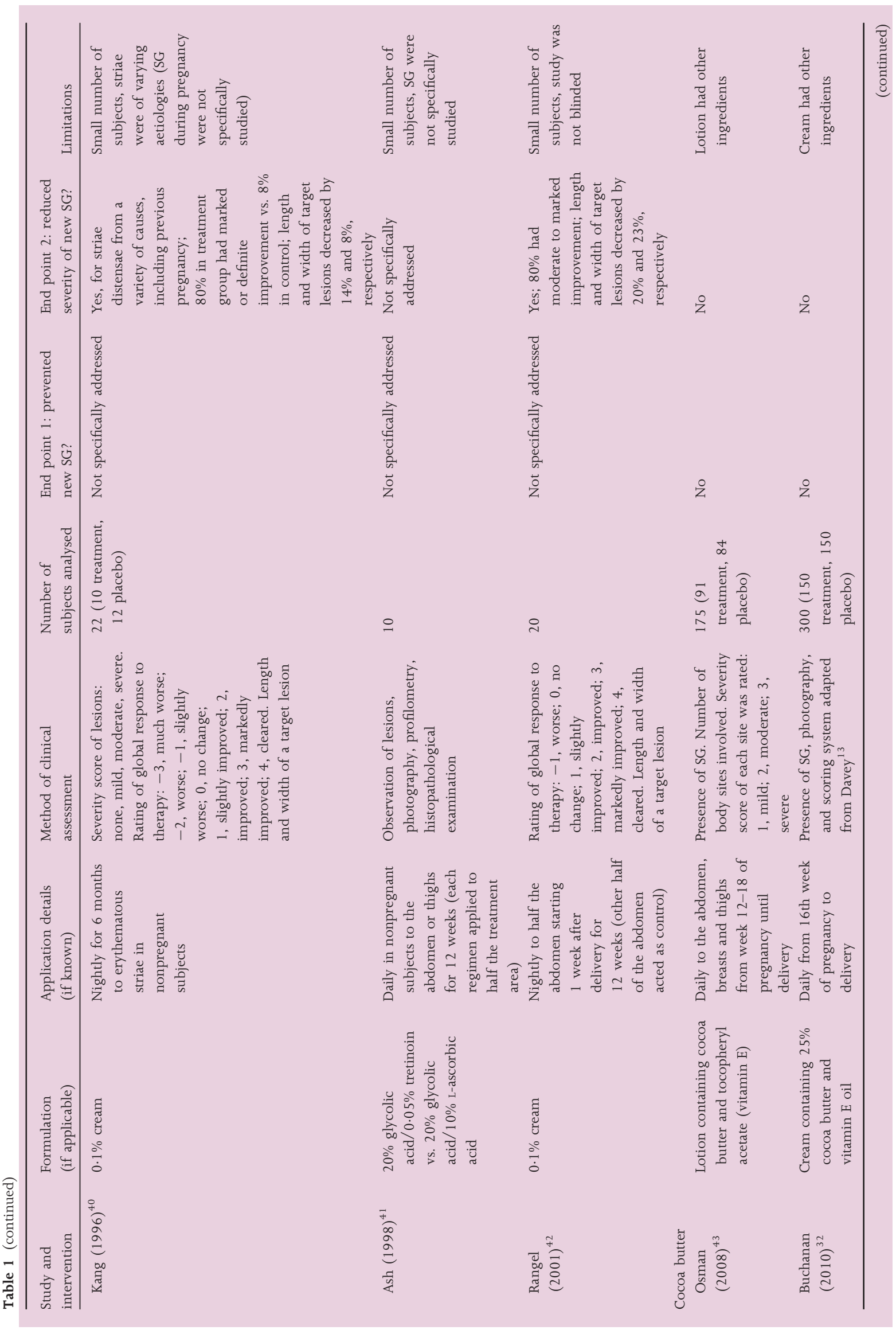




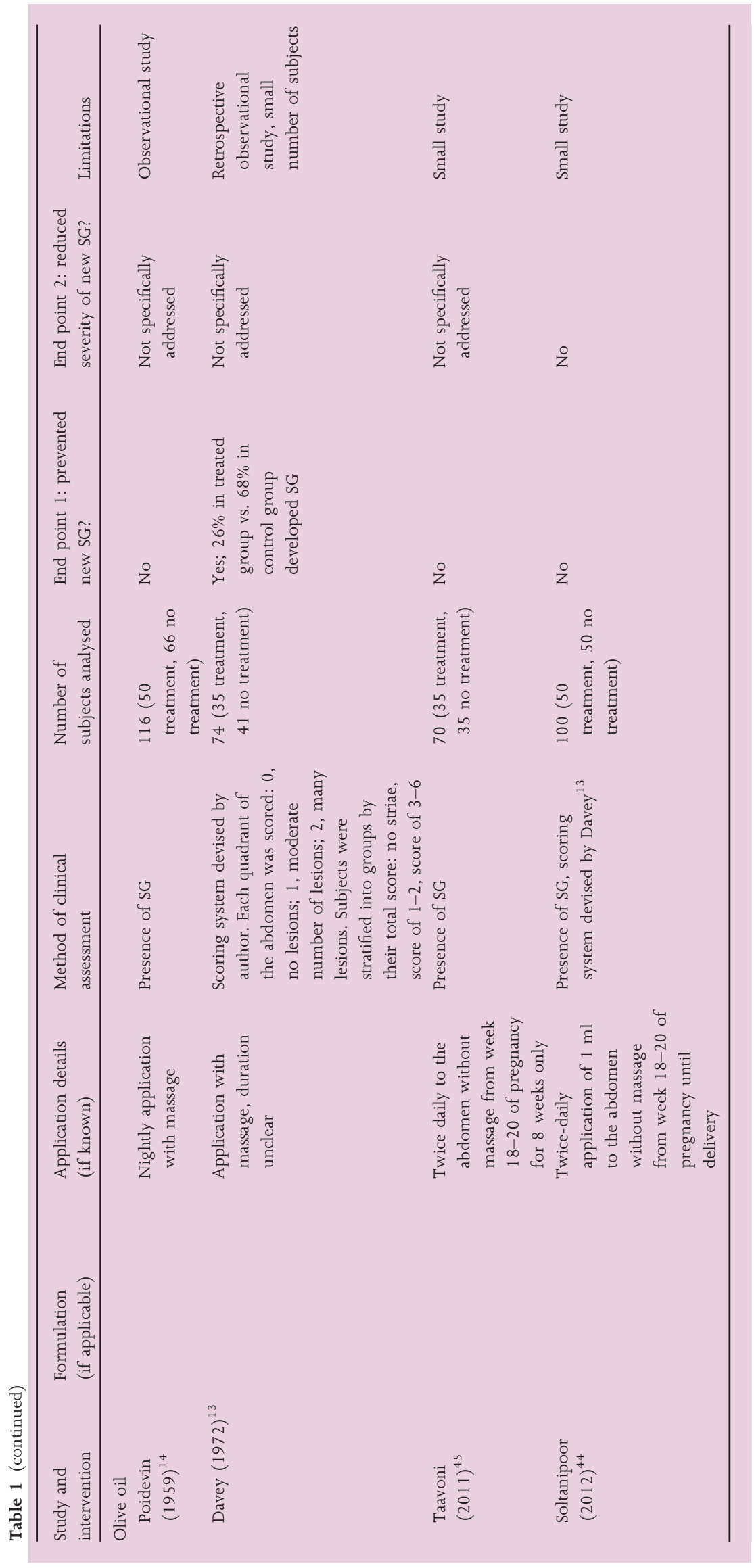


Table 2 Risk factors potentially associated with development of striae gravidarum

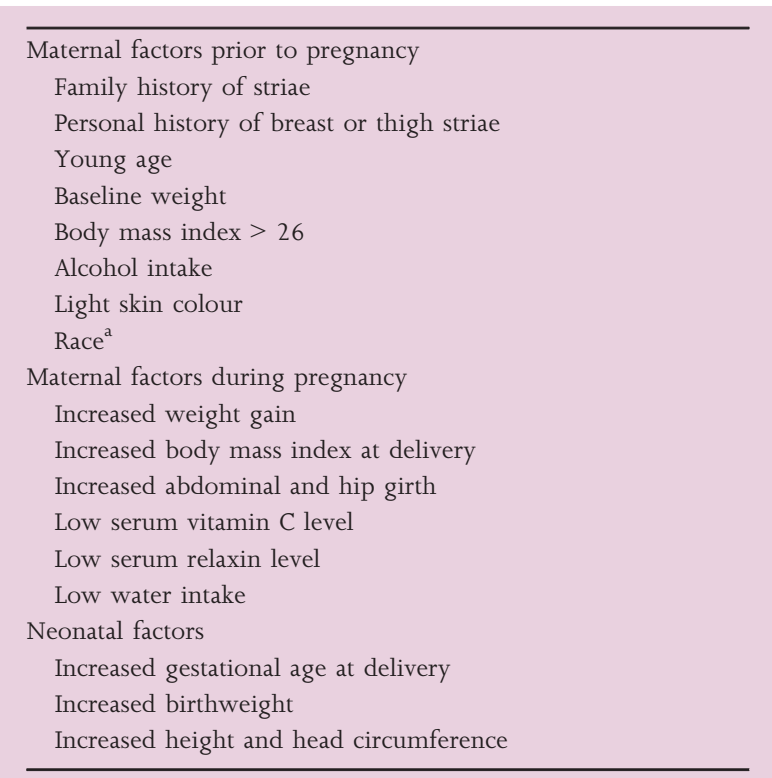

a Depending on the study, both nonwhite and white race have been associated with increased risk.

Topical application accelerates wound healing and improves the tensile strength of scars. ${ }^{28}$ It is possible that centella may improve SG by similar means, as lesions demonstrate clinical and histological features that overlap with scars. ${ }^{29}$

In a randomized, double-blind, placebo-controlled trial involving 80 women, Mallol et al. showed that daily massage with Trofolastin cream to the abdomen, breasts, buttocks and hips from the 12th week of pregnancy until delivery was associated with decreased SG incidence. ${ }^{1,30}$ Whereas 56\% (22 of 39) of women in the placebo group developed SG, 34\% (14 of 41) in the treatment group developed lesions. The cream was effective for preventing SG in subjects with a history of puberty-associated SD, but not in subjects with a history of SG from preceding pregnancies. In subjects who developed SG, the cream reduced the severity of lesions, compared with placebo.

In a randomized, double-blind, placebo-controlled trial, García Hernández et al. enrolled 183 women, who applied the cream containing centella triterpenes twice a day to the abdomen, thighs, hips, buttocks and breasts starting in the 12th week of pregnancy. ${ }^{31}$ The incidence of SG was similar between the treatment and placebo groups (38\% vs. $33 \%$, respectively). However, among women who developed SG, the severity of lesions remained stable with treatment, while worsening with placebo. In women without a history of SD, $6 \%$ of subjects (one of 17) undergoing treatment developed SG, compared with $35 \%$ (seven of 20) in the control arm. The authors concluded that the cream reduced the severity of SG developing during pregnancy, while decreasing the incidence of new SG in women without a history of SD.

Centella is thought to be the active agent in these creams. ${ }^{1}$ As this ingredient is often combined with others, well-designed controlled studies are required to determine the specific efficacy of centella for SG prevention. This ingredient may not be readily available in certain countries. ${ }^{32}$ In the U.S.A. it is available over the counter and potentially costly.

\section{Almond oil}

- There is weak evidence that massage with bitter almond oil may be effective for preventing SG and reducing their severity.

- Other products containing almond oil have not shown similar benefit.

Oils have moisturizing properties, and massage may stimulate blood flow to the skin. ${ }^{33}$ It is unclear how the combination of massage with oils may prevent SG or lessen their severity. Bitter almond oil has been used for SG because it reportedly does not pose a risk to the mother and foetus during pregnancy. ${ }^{33}$

In a nonrandomized, comparative study of 141 women with no history of SG, Timur Tashan and Kafkasli divided subjects into three groups. ${ }^{33}$ The first group applied bitter almond oil with a 15-min massage every other day during weeks 19-32 of pregnancy, followed by daily application until delivery. The second group applied the oil identically without massage. The third applied nothing. The development of abdominal SG was significantly reduced in the oil plus massage group (16 of 47), compared with oil alone (31 of 48) and the control group (33 of 46). Additionally, if women had developed SG in the abdominal area, lesions were less severe in the oil plus massage group. As bitter almond oil alone was ineffective and massage alone was not studied, further investigation is necessary to clarify which component may be more beneficial for preventing SG. Other studies discussed here have suggested that massage, along with emollients/creams, may be effective for preventing SG. ${ }^{1,34}$

In a more recent randomized controlled trial, almond oil was ineffective as part of a proprietary cream (Saj) containing lanolin, stearin, triethanolamine and bizovax glycerin amidine. ${ }^{35}$ Soltanipour et al. analysed 150 nulliparous women in the second trimester and observed no difference in the incidence or severity of abdominal SG between no intervention, olive oil without massage, or Saj cream without massage (50 subjects in each group). Lesions developed in 60\%, 64\% and $64 \%$ of participants, respectively.

\section{Hyaluronic acid}

- There is weak evidence that creams containing hyaluronic acid, including Alphastria and Verum, can prevent SG.

Alphastria is a proprietary cream containing hyaluronic acid, allantoin, vitamin A, vitamin $\mathrm{E}$ and calcium pantothenate. Verum is another proprietary cream containing vitamin E, essential fatty acids, panthenol, hyaluronic acid, elastin and menthol. The active ingredient in both is hyaluronic acid, which is thought to improve tensile resistance to mechanical 
forces and counteract atrophy by stimulating fibroblast activity and collagen production to increase skin volume. ${ }^{25}$ The exact mechanism of hyaluronic acid in preventing SG is unclear.

Two small studies suggest that hyaluronic acid-containing compounds may prevent SG during pregnancy. In a doubleblind study involving pregnant women, de Buman et al. demonstrated that Alphastria cream reduced the incidence of SG, compared with placebo (three of 30 vs. 11 of 30 subjects, respectively). ${ }^{36}$

Wierrani et al. enrolled 50 pregnant women at 20 weeks' gestation, and found that SG developed in 29\% (seven of 24) of women who applied Verum to the abdomen, thighs and breasts with massage, compared with $62 \%$ of control subjects (16 of 26), who performed no massage or topical application. ${ }^{34}$ This study was not placebo controlled and was poorly randomized. ${ }^{30}$ Parity of participants was also unclear, making it difficult to ascertain whether participants had SG from earlier pregnancies. ${ }^{37}$ Therefore, further investigation of this preparation for SG prevention is needed, and the specific effects of the ingredients vs. massage need clarification.

\section{Tretinoin}

- Tretinoin shows promise for decreasing the severity of erythematous SG.

- Tretinoin is a pregnancy category C drug, and therefore patients should wait until the postpregnancy/lactation period to apply it.

Topical tretinoin partially restores decreased collagen formation in photoaged skin by stimulating the synthetic activity of dermal fibroblasts. As damage of structural proteins such as collagen may also occur in SG, topical tretinoin has been studied for the treatment of this condition. In this context, its exact mechanisms remain unclear.

Early studies produced conflicting results. In an observational study by Elson, tretinoin led to 'significant improvement' of SD from a variety of causes in 15 of 16 patients. ${ }^{38}$ In a double-blind, placebo-controlled study by Pribanich et al., tretinoin cream $0.025 \%$ was applied daily for 7 months to abdominal SG at various stages of development, with six subjects assigned to treatment and five to placebo. ${ }^{39}$ No improvement occurred with treatment compared with placebo.

More recently, in a double-blind, randomized controlled study by Kang et al., 22 patients with early (erythematous) SD from a variety of causes, including pregnancy, applied a higher strength of tretinoin cream $(0 \cdot 1 \%)$ or vehicle nightly. ${ }^{40}$ After 6 months, eight of 10 subjects in the tretinoin group demonstrated marked or definite improvement of lesions, compared with one of 12 subjects in the vehicle group. Lesions treated with tretinoin showed a mean decrease in length and width by $14 \%$ and $8 \%$, respectively, whereas lesions treated with vehicle increased by $10 \%$ and $24 \%$, respectively. In a small study of 10 subjects by Ash et al., the appearance of mature white SD on the abdomen or thighs improved with either $20 \%$ glycolic acid/
$0.05 \%$ tretinoin or $20 \%$ glycolic acid $/ 10 \%$ L-ascorbic acid. ${ }^{41}$ Finally, in an open-label, prospective study by Rangel et al., tretinoin $0 \cdot 1 \%$ cream was applied nightly to pregnancy-related abdominal SG starting 1 week after delivery. ${ }^{42}$ After 12 weeks of application, 16 of 20 subjects demonstrated moderate to marked improvement, and the mean length and width of target lesions decreased by $20 \%$ and $23 \%$, respectively.

Of the studies discussed, only three enrolled subjects with pregnancy-related SG, ${ }^{39,40,42}$ and only one examined tretinoin use specifically for new-onset $\mathrm{SD} .{ }^{40}$ The most common sideeffects in the studies were localized erythema, scaling, itching and burning, all of which were mild and easily treated with emollients. Topical tretinoin is a pregnancy category $\mathrm{C}$ drug, and its use during pregnancy and lactation is not generally recommended.

\section{Cocoa butter}

- Studies suggest that cocoa butter, which is often combined with vitamin E, is not effective for preventing SG or reducing their severity.

Cocoa butter is a natural fat derived from cocoa beans, which grow on the tree Theobroma cacao. It is often combined with vitamin E oil. Some medical providers suggest applying topical cocoa butter before, during and after pregnancy to prevent SG development. ${ }^{43}$ Cocoa butter has emollient properties, although its mechanism of action is not known. Furthermore, the role of vitamin E in preventing SG is unclear.

In a double-blind, randomized, placebo-controlled trial, Osman et al. studied 175 nulliparous pregnant women, 91 of whom applied a lotion containing cocoa butter and vitamin $\mathrm{E}$ daily to the abdomen, breasts and thighs from 12-18 weeks' gestation until delivery. ${ }^{43}$ The remaining subjects applied a placebo lotion lacking cocoa butter and vitamin E. There was no difference in SG development between the intervention and control groups. If SG developed, the severity of lesions was similar between the two groups.

Buchanan et al. conducted a similar randomized, doubleblind, placebo-controlled study in 300 pregnant AfroCaribbean women with no previous history of SD. ${ }^{32}$ Of these subjects, 150 applied a cream containing 25\% cocoa butter and vitamin E oil daily from 16 weeks' gestation to delivery, while the remaining applied a placebo cream. No statistically significant difference was noticed in the development of SG between the treatment and placebo groups, with lesions developing in $44 \%$ vs. $55 \%$ of subjects, respectively. The intervention cream did not reduce the severity of new SG compared with placebo.

\section{Olive oil}

- Studies suggest that olive oil is not effective for preventing SG or reducing their severity.

The use of olive oil for preventing SG is popular. Olive oil is rich in vitamin $\mathrm{E}$ and has emollient properties. ${ }^{44}$ 
Early research produced conflicting results. In an observational study of 116 primagravid women, Poidevin noted that 36 of 50 pregnant women who applied olive oil nightly developed abdominal SG, compared with 36 of 66 pregnant women who did not. ${ }^{14}$ These observations suggested that olive oil was not effective as prophylaxis for SG development. In another early observational study, Davey found that the incidence of SG was reduced by up to $42 \%$ in primiparous women who had used olive oil during pregnancy, compared with those who did not ( $26 \%$ vs. $68 \%$, respectively). ${ }^{13}$ The duration of olive oil use was unclear. These data were obtained retrospectively from questions asked of 76 women in the postpartum period and, therefore, are subject to recall bias.

In a more recent randomized controlled trial, Taavoni et al. studied the use of olive oil for a short duration in 70 nulliparous pregnant women. ${ }^{45}$ Participants at 18-20 weeks of gestation were randomized to no treatment or application of olive oil to the abdomen without massage twice a day for 8 weeks. Although the incidence of SG was $46 \%$ (16 of 35 ) in the intervention group vs. 63\% (22 of 35) in the control group, this difference was not statistically significant. The authors concluded that initiation of olive oil in the second trimester did not prevent SG onset.

In the second phase of the same randomized controlled trial, Soltanipoor et al. followed 100 nulliparous pregnant women, who applied either nothing or $1 \mathrm{ml}$ of olive oil to the abdomen without massage twice daily from 18-20 weeks' gestation until delivery. ${ }^{44}$ This intervention did not reduce the incidence or severity of SG. Overall, SG developed in 64\% (32 of 50) of the intervention group and $60 \%$ (30 of 50) of the control group.

\section{Recommendations}

There is limited evidence that centella and possibly massage with bitter almond oil may prevent SG and/or reduce the severity of lesions. There is weak evidence that hyaluronic acid can prevent SG. Tretinoin holds promise for reducing the severity of erythematous SG, but its use is limited by its pregnancy category. Cocoa butter and olive oil do not demonstrate efficacy for preventing SG or reducing their severity.

\section{Conclusions}

Reliable methods for preventing SG during pregnancy are lacking. ${ }^{30}$ Compounds that potentially prevent new-onset SG or reduce their severity lack evidence from rigorous, high-quality, well-designed, randomized controlled trials with ample subject numbers. ${ }^{37}$ Additionally, methods of assessing SG severity or response to therapy vary considerably among published studies (Table 1).

Importantly, SG can cause significant distress. ${ }^{2,12,15}$ Yet, major barriers exist to conducting rigorous research on SG prevention. For instance, the medical and scientific community remains reluctant to devote substantial research to this condition, likely because the lesions are not physically harmful. Accordingly, the pathogenesis of SG remains unclear, hampering the development of rational therapies. The development of lesions also remains difficult to predict, hindering subject recruitment for prospective studies. In this respect, high-risk groups that tend to develop lesions in predictable anatomical locations, such as pregnant women, may not only be a suitable population for SD prevention trials, but also stand to benefit greatly from positive findings.

Additionally, numerous questions remain regarding the use of prophylactic agents for SG during pregnancy. For instance, if a topical agent is initiated for prophylaxis against SG development, when should a pregnant woman begin to use it? How long should she use it? If a topical agent is used to reduce the severity of recently developed SG, how soon should treatment commence after lesions have formed? Does the age of the subject or multiple gestations impact the efficacy of topical agents? Do combinations of the topical agents discussed above hold promise? What is the safety of various topical modalities during pregnancy and lactation? Finally, what is the cost-benefit ratio of preventative therapies used for SG? These concerns will be important to address in future studies.

\section{References}

1 Mallol J, Belda MA, Costa D et al. Prophylaxis of striae gravidarum with a topical formulation. A double blind trial. Int J Cosmet Sci 1991; 13:51-7.

2 Salter SA, Kimball AB. Striae gravidarum. Clin Dermatol 2006; 24:97-100.

3 Al-Himdani S, Ud-Din S, Gilmore S, Bayat A. Striae distensae: a comprehensive review and evidence-based evaluation of prophylaxis and treatment. Br J Dermatol 2014; 170:527-47.

4 Chang AL, Agredano YZ, Kimball AB. Risk factors associated with striae gravidarum. J Am Acad Dermatol 2004; 51:881-5.

5 Shuster S. The cause of striae distensae. Acta Derm Venereol Suppl (Stockh) 1979; 59:161-9.

6 Zheng P, Lavker RM, Kligman AM. Anatomy of striae. Br J Dermatol 1985; 112:185-93.

7 Thomas RG, Liston WA. Clinical associations of striae gravidarum. J Obstet Gynaecol 2004; 24:270-1.

8 Sheu HM, Yu HS, Chang $\mathrm{CH}$. Mast cell degranulation and elastolysis in the early stage of striae distensae. J Cutan Pathol 1991; 18:410-16.

9 Watson RE, Parry EJ, Humphries JD et al. Fibrillin microfibrils are reduced in skin exhibiting striae distensae. Br J Dermatol 1998; 138:931-7.

10 Tsuji T, Sawabe M. Elastic fibers in striae distensae. J Cutan Pathol $1988 ; 15: 215-22$.

11 Mitts TF, Jimenez F, Hinek A. Skin biopsy analysis reveals predisposition to stretch mark formation. Aesthet Surg J 2005; 25:593600 .

12 Yamaguchi K, Suganuma N, Ohashi K. Quality of life evaluation in Japanese pregnant women with striae gravidarum: a cross-sectional study. BMC Res Notes 2012; 5:450.

13 Davey CM. Factors associated with the occurrence of striae gravidarum. J Obstet Gynaecol Br Commonw 1972; 79:1113-14.

14 Poidevin LO. Striae gravidarum. Their relation to adrenal cortical hyperfunction. Lancet 1959; 2:436-9. 
15 Madlon-Kay DJ. Striae gravidarum. Folklore and fact. Arch Fam Med 1993; 2:507-11.

16 Atwal GS, Manku LK, Griffiths CE, Polson DW. Striae gravidarum in primiparae. Br J Dermatol 2006; 155:965-9.

17 Ghasemi A, Gorouhi F, Rashighi-Firoozabadi M et al. Striae gravidarum: associated factors. J Eur Acad Dermatol Venereol 2007; 21:7436.

18 J-Orh R, Titapant V, Chuenwattana P, Tontisirin P. Prevalence and associate factors for striae gravidarum. J Med Assoc Thai 2008; 91:445-51.

19 Osman H, Rubeiz N, Tamim H, Nassar AH. Risk factors for the development of striae gravidarum. Am J Obstet Gynecol 2007; 196:62 e1-5.

20 Findik RB, Hascelik NK, Akin KO et al. Striae gravidarum, vitamin C and other related factors. Int J Vitam Nutr Res $2011 ; \mathbf{8 1 : 4 3 - 8 .}$

21 Lurie S, Matas Z, Fux A et al. Association of serum relaxin with striae gravidarum in pregnant women. Arch Gynecol Obstet 2011; 283:219-22.

22 Salter SA, Batra RS, Rohrer TE et al. Striae and pelvic relaxation: two disorders of connective tissue with a strong association. J Invest Dermatol 2006; 126:1745-8.

23 Lerdpienpitayakul R, Manusirivithaya S, Wiriyasirivaj B et al. Prevalence and risk factors of striae gravidarum in primiparae. Thai $J$ Obstet Gynaecol 2009; 17:70-9.

24 Schwingel AC, Shimura Y, Nataka Y et al. Exercise and striae distensae in obese women. Med Sci Sports Exerc 2003; 35 (Suppl.):S33.

25 Elsaie ML, Baumann LS, Elsaaiee LT. Striae distensae (stretch marks) and different modalities of therapy: an update. Dermatol Surg 2009; 35:563-73.

26 Gohil KJ, Patel JA, Gajjar AK. Pharmacological review on Centella asiatica: a potential herbal cure-all. Indian J Pharm Sci 2010; 72:54656.

27 Velasco M, Romero E. Drug interaction between asiaticoside and some anti-inflammatory drugs in wound healing of the rat. Curr Ther Res Clin Exp 1976; 19:121-5.

28 Brinkhaus B, Lindner M, Schuppan D, Hahn EG. Chemical, pharmacological and clinical profile of the East Asian medical plant Centella asiatica. Phytomedicine 2000; 7:427-48.

29 Arem AJ, Kischer CW. Analysis of striae. Plast Reconstr Surg 1980; 65:22-9.

30 Young GL, Jewell D. Creams for preventing stretch marks in pregnancy. Cochrane Database Syst Rev 2000: CD000066.

31 García Hernández JÁ, Madera González D, Padilla Castillo M, Figueras Falcón T. Use of a specific anti-stretch mark cream for preventing or reducing the severity of striae gravidarum.
Randomized, double-blind, controlled trial. Int J Cosmet Sci 2013; 35:233-7.

32 Buchanan K, Fletcher HM, Reid M. Prevention of striae gravidarum with cocoa butter cream. Int J Gynaecol Obstet 2010; 108:65-8.

33 Timur Tashan S, Kafkasli A. The effect of bitter almond oil and massaging on striae gravidarum in primiparaous women. J Clin Nurs 2012; 21:1570-6.

34 Wierrani F, Kozak W, Schramm W, Grünberger W. [Attempt of preventive treatment of striae gravidarum using preventive massage ointment administration]. Wien Klin Wochenschr 1992; 104:424 (in German).

35 Soltanipour F, Delaram M, Taavoni S, Haghani H. The effect of olive oil and the $\mathrm{Saj}^{\circledR}{ }^{\circledR}$ cream in prevention of striae gravidarum: a randomized controlled clinical trial. Complement Ther Med 2014; 22:220-5.

36 de Buman M, Walther M, de Weck R. [Effectiveness of Alphastria cream in the prevention of pregnancy stretch marks (striae distensae). Results of a double-blind study]. Gynakol Rundsch 1987; 27:79-84 (in German).

37 Brennan M, Young G, Devane D. Topical preparations for preventing stretch marks in pregnancy. Cochrane Database Syst Rev 2012; 11: CD000066.

38 Elson ML. Treatment of striae distensae with topical tretinoin. J Dermatol Surg Oncol 1990; 16:267-70.

39 Pribanich S, Simpson FG, Held B et al. Low-dose tretinoin does not improve striae distensae: a double-blind, placebo-controlled study. Cutis 1994; 54:121-4.

40 Kang S, Kim KJ, Griffiths CE et al. Topical tretinoin (retinoic acid) improves early stretch marks. Arch Dermatol 1996; 132:519-26.

41 Ash K, Lord J, Zukowski M, McDaniel DH. Comparison of topical therapy for striae alba (20\% glycolic acid/0.05\% tretinoin versus $20 \%$ glycolic acid $/ 10 \%$ L-ascorbic acid). Dermatol Surg 1998; 24:849-56.

42 Rangel O, Arias I, García E, Lopez-Padilla S. Topical tretinoin 0.1\% for pregnancy-related abdominal striae: an open-label, multicenter, prospective study. Adv Ther 2001; 18:181-6.

43 Osman H, Usta IM, Rubeiz N et al. Cocoa butter lotion for prevention of striae gravidarum: a double-blind, randomised and placebo-controlled trial. BJOG 2008; 115:1138-42.

44 Soltanipoor F, Delaram M, Taavoni S, Haghani H. The effect of olive oil on prevention of striae gravidarum: a randomized controlled clinical trial. Complement Ther Med 2012; 20:263-6.

45 Taavoni S, Soltanipour F, Haghani H et al. Effects of olive oil on striae gravidarum in the second trimester of pregnancy. Complement Ther Clin Pract 2011; 17:167-9. 